\title{
Forensic evidence
}

Fresh career opportunities could develop in forensic science, if recommendations in a report from the US National Research Council are adopted, says forensic scientist and co-author Jay Siegel.

Forensic scientists need to prove their competence with recognized qualifications at different levels, says Strengthening Forensic Science in the United States: A Path Forward. Concerned members of Congress had asked the National Academy of Sciences to propose reforms that would coordinate and improve forensic-science analyses across federal, state and local jurisdictions. The report recommends mandatory certification for the pathologists, biologists, physicists, chemists and medical officers working in forensics.

To set these rigorous standards for the field, it calls for the creation of an independent National Institute of Forensic Science. Without such an institute, says report co-chair Constantine Gatsonis, a biostatistician at Brown University in Providence, Rhode Island, forensic science will continue to lack the funds needed to mature the field.

More thorough scientific evaluation of forensic protocols may generate new jobs, predicts Siegel, director of the forensic and investigative sciences programme at Purdue University in Indianapolis, Indiana. "The biggest problem in forensic science is a lack of science-based research to settle what can be considered evidence in the courtroom." For example, he says, despite the routine acceptance of fingerprints in the courts, evidence is still lacking as to how well a given fingerprint identifies a specific person.

Siegel believes that if Congress adopts some of the recommendations, the field will experience a hiring boom when the economy recovers. "There is a tremendous pent-up need for new scientists," says Siegel. A 2005 survey, Census of Publicly Funded Forensic Crime Laboratories, 2002, of crime-lab directors indicated 1,900 additional forensic scientists were needed to get case management down to the desired 30-day turnaround. And,

Siegel says, staffing needs have only increased since then.

At present, certification programmes for individuals and accreditation of education programmes and crime laboratories are voluntary. However, these are not all supervised by the American Academy of Forensic Sciences (AAFS), which has spent the past decade establishing a board to examine the certifying bodies in current existence. Although AAFS president Thomas Bohan agrees that certification is important, he thinks the academy's existing system is sufficient. He believes that the report's emphasis on certification will prod most forensic scientists and institutions to flock to AAFS-approved certifying boards, making a new overseeing body unnecessarily complicated.

The recommendations could also push more forensic-science educational programmes to seek accreditation. Of the roughly 200 now operating, according to AAFS, only 19 are accredited by its Forensic Science Education Programs Accreditation Commission. Virginia Gewin

\section{Possooc ouvenat \\ Job juggling}

My wedding celebration is over, the flights booked, my visa nestled in my passport. Now all I have to do is complete all the projects I'm working on before I leave Australia to spend the next two years in the United States. Since my postdoc contract ended last year, I have been paying the bills by working on three part-time projects that add up to a full-time workload. During an average day I juggle my time between them from examining the impacts of dingos on Australia's mammals, to writing a book chapter on the impacts of climate change on Western Australian biodiversity, to writing website content for a new national climate-change research network.

I am grateful for the work, and dependent on the money it brings, but I yearn to do my own research. As I struggle to find enough hours in the day, unfinished manuscripts sit forlornly in a folder on my desktop. Others wait for me to address reviewer comments and resubmit them to journals This does not bode well for my 2009 publication record.

With our forthcoming move to the United States, my

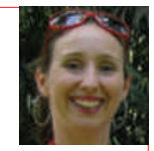

husband working full time, and a toddler to care for, I can't see this cycle of part-time work ending any time soon. So perhaps I should embrace it rather than fight it.

Indeed, the benefits are many. I get the opportunity to work on a diverse range of interesting projects, and the flexible hours allow me more time with my son. And maybe one day l'll embrace those lonely manuscripts and finish them once and for all. Joanne Isaac was a postdoc in climate-change effects on biodiversity at James Cook University, Townsville, Australia.

\section{IN BRIEF}

\section{Drug firms cut back}

Several drug research companies across North America, including five US-based firms and a Canadian biotech, have announced lay-offs.

Hospira of Lake Forest, Illinois, a pharmaceutical and medication delivery firm specializing in injectable drugs, will cut about 1,400 employees, or $10 \%$ of its global workforce. Cortex Pharmaceuticals of Irvine, California, which makes drugs to treat psychiatric and nervous-system disorders, cut 14 of 27 employees.

Poniard Pharmaceuticals of South San Francisco, California, is cutting eight of its 67 employees, discontinuing in-house preclinical research and focusing on picoplatin, a next-generation platinum chemotherapy. Adventrx Pharmaceuticals of San Diego, California, is cutting its payroll to five and is discontinuing drug-development efforts and business operations to focus on "strategic options". In December 2008 the company employed about 35 people, according to its website.

Synta Pharmaceuticals of Lexington, Massachusetts, cut 90 positions from its 220-member workforce owing to unfavourable late-stage clinical-trial results on a metastatic melanoma treatment. Synta has five programmes in clinical or preclinical development and several others in the discovery stage. Canada's Bellus Health is cutting its staff by nearly half. It did not report exact numbers, but the company employed 170 in December 2007, according to its website.

\section{Syngene centre opens}

Syngene International, a subsidiary of Indian biotech Biocon, and US drugmaker Bristol-Myers Squibb (BMS) have opened a research-and-development centre in Bangalore.

The 18,000-square-metre facility, which employs 270 researchers, helps advance BMS's discovery and early drug-development efforts. It will house 360 researchers by the end of the year and plans to ramp that number up to 450 .

Work at the facility will span the drug discovery and development process. Construction began in March 2007, when BMS and Biocon agreed to focus on integrated drug discovery and development capabilities at Syngene. 